\section{Defining remission and treatment success using the DAPSA score: response to letter by Helliwell and Coates}

We truly appreciate the comments on our study of Disease Activity Index for Psoriatic Arthritis (DAPSA) disease activity states and DAPSA responses provided by Drs. Helliwell and Coates, ${ }^{1}$ because this gives us a dual opportunity: first, to address the principal differences in our thinking on psoriatic arthritis (PsA) compared with that of the authors of the letter, and second to expand on our findings. The authors, whose work we have followed and valued highly for many years, present a large number of comments that we will try to answer individually.

1. Concerns about only assessing joints. The authors ought to take notice that the DAPSA is not solely comprised of joint counts, but, aside from C-reactive protein (CRP), also includes patient global assessment (PtGA) as well as patient pain assessment $(\mathrm{PtP})$.

2. Cut-offs were developed based entirely on physician opinion without including patients. While we sent our survey only to physicians, it needs to be borne in mind that the DAPSA includes PtGA and PtP and thus is already heavily weighted with the views of the patient. This item is again mentioned as being 'disappointing' toward the end of the letter. However, we were partly inspired by the authors themselves who stated in their publication on the definition of moderate disease activity (MDA) that the "questionnaire ... was then circulated to rheumatologists and dermatologists with an interest in PsA, identified through membership of GRAPPA", ${ }^{2}$ without involving patients. To us it seemed preferable to obtain the patients' opinions on cut points and responses in a separate currently evolving activity. This will enable involving a large number of patients, rather than adding a few patients to the survey as suggested by the authors.

3. Response criteria used randomised controlled trial (RCT) datasets rather than data from the PsA population as a whole. In contrast to the authors, we feel that it is best to derive response criteria from RCTs rather than use observational data, for the following reasons: (i) RCTs are multicentre studies and thus comprise a much broader population of patients than seen in a single centre or a single country; (ii) RCTs require a minimum level of disease activity and thus allow the generation of contrasts, while observational data too often struggle from the inclusion of patients with low disease activity, in whom improvements are difficult to see; (iii) RCTs collect data in a predefined and usually complete way, while practice populations may frequently lack important variables or values; and (iv) evaluations in RCTs are based on consistently spaced follow-up examinations, while in observational data the time between examinations may vary dramatically and data require extrapolation; there are many more reasons to prefer RCT data, which we will not elude to for the sake of space. Nevertheless, we agree with the authors that data derived from RCT cohorts need to be validated in real-life patients. To this end we would like to draw their attention to the fact that both the DAPSA as well as the DAPSA response criteria were validated in our observational dataset. $^{34}$

4. The DAPSA does not cover all domains designated by the GRAPPA/OMERACT group as it does not incorporate psoriasis, function and quality of life. This criticism has to be addressed in several ways because it reveals some fundamental conceptual differences that distinguish our views from those of the authors. First, the authors are reminded that we adopted the DAPSA after performing a principal component analysis using all variables that achieved over $50 \%$ of the votes of the OMERACT group. The authors are referred to this publication, ${ }^{5}$ which revealed that pain, joints and acute phase reactants were the most appropriate components among the OMERACT disease activity variables. In this analysis, skin was a separate component loading on a non-significant factor. Other OMERACT variables, such as enthesitis, dactylitis or spinal involvement, constituted redundancies of the major components that are actually represented in the DAPSA. Second, we purposely refrained from including psoriasis in the DAPSA, the definitions of disease activity states or response criteria, because skin involvement was not a significant factor related to disease activity in the above-mentioned principal component analysis; and because we do not feel that it is justified to amalgamate skin and joint disease into a single score as long as it is not proven that both are due to the same pathogenetic mechanisms. And third, while the authors appear to regard physical function and quality of life as a reflection of disease activity, we do not consider them as disease activity measures for reasons that we have repeatedly eluded to in discussion of rheumatoid arthritis (RA): (i) physical function in arthritis comprises reversible and irreversible components with the reversible component being highly related to disease activity (ie, assessed by the DAPSA) and the irreversible component being highly related to joint damage ${ }^{67}$ (which is not a major component of the GRAPPA/OMERACT set having received the lowest score $^{8}$ ); as long as this conclusion is not refuted it has to be accepted as likely also being valid in PsA; (ii) function and quality of life can be dramatically impaired by comorbidities ${ }^{9}{ }^{10}$ which also in PsA are a consequence rather than the cause of the disease ${ }^{11}$ thus, we have no reason to doubt that impairment of function and quality of life due to comorbidities is also part of PsA; (iii) in the principal component analysis, the Health Assessment Questionnaire (HAQ) and the Medical Outcome Study Short Form-36 (SF-36) exhibited lower values than the chosen variables in both the joint and the pain components. For all these reasons, function and quality of life are at least as much outcomes as process variables and therefore should not be included in a composite measure but assessed in their own right. The authors will hopefully acknowledge that function and quality of life can be very bad due to past joint damage and inflammatory disease activity still be in remissionagain: in our study we speak of remission of inflammatory activity and not of remission of all aspects of the disease, or the absence of comorbidities.

5. In our survey doctors were only given information on the variables contained in the DAPSA and not on entheseal, axial and skin involvement which could radically affect expert classification. This point is absolutely correct. This step was deliberate, since we wanted to learn about the experts' opinion on arthritis activity and did not want to see this 'radically' influenced by variables that are not related to joint disease, given that we do not know which of these other aspects has the same pathogenetic background. Using this approach, we could readily classify the disease activity states and response criteria of joint involvement rather than being confounded by other aspects of the disease. 
6. High frequency of enthesitis. The authors have a point in that the frequency of enthesitis is, indeed, variable and may be higher than we suggested, although a recent Canadian observational database reported that just $14 \%$ of patients had enthesitis. ${ }^{12}$ However, this was neither the main nor the only argument that we brought forward in the specific sentence, rather-and this is the main point-there are separate instruments for assessing enthesitis and we suggest those that are pertinent should be evaluated rather than enthesitis amalgamated into an overall score. Also, as we clearly stated, enthesitis will be reflected in patients' assessments.

7. The remission cut point of 4 could miss active joint disease and lead to dangerous undertreatment in a treat-to-target strategy. Indeed, the remission cut point of 4 may allow for some residual activity. However, given the five components of the DAPSA, it is highly unlikely that more than one or two swollen joints would be present, a status easily compatible with the definition of remission as "absence ... of significant inflammatory disease activity". ${ }^{13}$ The authors' worry is also dismissed in a study by Husic et al who showed that DAPSA remission (using a cut point close to that determined here) had much higher specificity for sonographic remission than MDA or the Composite Psoriatic Disease Activity Index (CPDAI). ${ }^{14}$ Moreover, independently of our study, Salaffi et $a l^{15}$ found a DAPSA score of 4 to be a cut point for remission and the specificity of the DAPSA to be almost $90 \%$, higher than that of the CPDAI or the Psoriatic Arthritis Disease Activity Score (PASDAS). All these independent data contradict the authors' theoretical concerns. With respect to implying "dangerous undertreatment", the authors may wish to refer to their own 'minimal disease activity state' (MDA) which requires five of seven components to reach the predefined cut point and thus may theoretically allow even very high residual joint activity plus another major activity problem to be called 'minimal' PsA activity (being a Tight Control of Psoriatic Arthritis (TICOPA) treatment target). ${ }^{2}$ While we do not believe this is the case, we simply mention it here to raise the authors' self-reflection, since, according to the definition, it is more likely to have high residual joint activity in MDA than it is in DAPSA remission.

8. Statistical advantage of composite measures in the development of a definition of remission. The DAPSA is a composite measure and thus has all the statistical advantages of a composite measure. ${ }^{16}{ }^{17}$ However, in accordance with the principal component analysis mentioned above, the components of this composite measure relate to the joint domain. There is no evidence that composite measures that comprise different domains which possibly respond differently to therapy are of any advantage. In this respect we would like to quote the authors themselves from a paper on remission in PsA: "There are, however, disadvantages with developing a composite score: a single score may underestimate improvements in some domains and deterioration in others that may be of importance in RCT of therapies that improve disease domains in different ways. Examples include agents that may improve skin manifestations more than articular ones, or those that benefit peripheral joints but not spinal manifestations or enthesitis". ${ }^{18}$ We could not agree more-and this is exactly what we suggest to be the appropriate way of dealing with PsA. Indeed, many compounds have shown dissociation of the cutaneous and joint activity responses.
9. Suggesting additional measures should be used for additional manifestations implies recommending the CPDAI or PASDAS. We are not sure how the authors arrive at this conclusion. On the contrary, we have suggested-and all of the reasons given above are supported by evidence-that one should assess arthritis separately from other domains and assess domain-specific scores when these domains are affected or it is worthwhile to assess them. In our view, lumping different disease characteristics together is not helpful and there is currently no evidence that this is necessary. Our desire was to provide an arthritis score for rheumatologists who can each decide if she or he wishes to evaluate additional domains of the disease. Importantly, as the authors agree in their introductory statement, the DAPSA is very easy to calculate. In contrast, the PASDAS requires a calculator and is heavily transformed and weighted, while the CPDAI also takes significant effortand all that without any added benefit in PsA, on the contrary. $^{14} 19$ These multi-organ-composite measures are impractical in daily life. In daily routine we practice medicine according to the patient's needs-why then should we gather all of this into one single score? If all these clinical features are based on different pathogenetic pathways, why should we use a composite measure that comprises some features that may not respond to a given treatment? Indeed, there is recent strong support from studies on genetics that clearly reveal that different clinical manifestations of PsA are associated with different genetic backgrounds. ${ }^{20} 21$ But even if they were all due to an identical pathomechanism, we feel that it is of the utmost importance to have a separate measure for arthritis: 'personalised' medicine is a commonly used term these days. Why not start by using measures that are personalised to each patient's needs, instead of taking a generic approach to patients with this complex heterogeneous disease without considering their presentation?

10. Individual components of PASDAS and CPDAI could be reported to capture different domains when evaluating therapies. This is an argument that we do not understand: why should anyone in trials or clinic go through the calculation of PASDAS and CPDAI in order to then separate the individual domains, which are truncated or modified version of other measures? For each of the disease's domains we have excellent validated instruments-should this not suffice? In our view, this point in itself raised by the authors themselves dismantles their concept and makes any assessment even more difficult. Along this line, we recently read a report that "of the specific PsA indices evaluated, CPDAI showed the poorest correlation with all the other activity measurements". ${ }^{22}$ And finally, related to the expected association of disease activity with cardiovascular risk, a recent study revealed that time integrated DAPSA, but not time integrated PASDAS, was associated significantly with the extent of atherosclerosis ${ }^{23}$ - do we need much more evidence that a measure of joint activity like the DAPSA is at least as good as a composite measure comprising all domains of psoriatic disease (if not better)? It has been shown consistently that PtGA is related to the joint domain and to other domains of the disease in general. ${ }^{12} 24$ Moreover, while such data are lacking in PsA, it has been revealed for juvenile inflammatory arthritis that patient pain assessment correlates highly with enthesitis. Thus, contrasting with the authors' contention, the DAPSA is a composite score comprising measures that also encompass other domains of the overall disease. 
11. Do the cutoffs have prognostic impact? This question is very pertinent and we are currently evaluating the correlation between disease DAPSA changes and outcome variables.

12. The use of ACR20/50/70 for developing the DAPSA response criteria. American College of Rheumatology (ACR) response criteria have been used and thus validated in all major PsA clinical trials. In their wonderful TICOPA trial, even the authors themselves have proven the validity of these response criteria and the significant difference in ACR response rates between a treat-to-target strategy and routine care. ${ }^{25}$ We applied these criteria as an anchor because we had several hundred patients with these outcomes available. If we had used physicians' ratings of major, minor or moderate improvement as an anchor, how many patient vignettes could we have used in a survey? And would the authors then not challenge the fact that we did not provide data on skin, entheses, dactylitis, etc? In the clinical trials all these data were available to patients and investigators and their global assessments attributed with this complete information. Moreover, the ACR response criteria, in contrast to the 28-joint Disease Activity Score (DAS28), use a complete and not a reduced joint count. Thus, we feel that it was an asset to have used these criteria. Why not use only ACR criteria? Because they do not constitute a continuous measure, do not allow assessment of actual disease activity, and are thus clearly of limited use in clinical practice.

Thus, we believe that we have been able to provide trialists and clinicians interested in PsA with a highly valid tool for disease activity assessment and evaluation of disease activity states. All of us agree that PsA is a heterogeneous disease, but we here feel that heterogeneity should not be accounted for by forcing it into a homogenising score. In contrast to other instruments, the DAPSA is a continuous instrument that can be used to assess PsA activity, while specific tools for other manifestations of psoriatic disease can and should be used in patients presenting with the respective features. We would also like to point out that assessment of ankylosing spondylitis also focuses on the spine, for instance by using the Bath Ankylosing Spondylitis Disease Activity Index (BASDAI) or the Ankylosing Spondylitis Disease Activity Score (ASDAS), and scientists working in this area do not lump peripheral arthritis, uveitis, enteritis, urethritis, enthesitis, etc into one score. Needless to say, the ankylosing spondylitis field has advanced dramatically in recent years despite this focus on the spine.

In conclusion, every single point of criticism raised by the authors of the letter has several counterpoints. The evidence is on the side of the DAPSA; it is valid and practicable in trials and in practice. For many years we have heard that finding criteria for remission in PsA may be elusive $e^{26}$ - now they are here. For many years response assessment was done by borrowing instruments from the RA field-now response criteria for PsA are available. We respect the authors very highly for all their contributions to the field of PsA, not least the TICOPA trial, but also for enhancing our state of knowledge on the complexity, the severity and the assessment modalities of PsA. In this light, rather than depreciate the value of our study, we would ask the authors to test the DAPSA in validation studies themselves. Needless to say, we will be very glad to collaborate with them to this end.

\footnotetext{
Monika M Schoels, ${ }^{1}$ Daniel Aletaha, ${ }^{2}$ Josef S Smolen ${ }^{2}$

${ }^{1}$ 2nd Department of Internal Medicine, Center for Rheumatic Diseases, Hietzing Hospital, Vienna, Austria

${ }^{2}$ Department of Rheumatology, Medical University of Vienna, Vienna, Austria
}

Correspondence to Dr Monika M Schoels, 2nd Department of Internal Medicine, Center for Rheumatic Diseases, Hietzing Hospital, Vienna 1130, Austria; monika. schoels@live.com

\section{Competing interests None declared.}

Provenance and peer review Commissioned; internally peer reviewed.

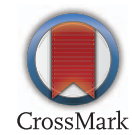

To cite Schoels MM, Aletaha D, Smolen JS. Ann Rheum Dis 2015;74:e67.

Received 18 September 2015

Accepted 19 September 2015

Published Online First 22 October 2015

\section{(S) Linked}

- http://dx.doi.org/10.1136/annrheumdis-2015-208509

Ann Rheum Dis 2015;74:e67. doi:10.1136/annrheumdis-2015-208521

\section{REFERENCES}

1 Helliwell P, Coates L. The definition of remission in psoriatic arthritis—can this be accurate without assessment of multiple domains? Ann Rheum Dis 2015;74:e66.

2 Coates LC, Fransen J, Helliwell PS. Defining minimal disease activity in psoriatic arthritis: a proposed objective target for treatment. Ann Rheum Dis 2010;69:48-53

3 Schoels M, Aletaha D, Funovits J, et al. Application of the DAREA/DAPSA score for assessment of disease activity in psoriatic arthritis. Ann Rheum Dis 2010;69:1441-7.

4 Schoels MM, Aletaha D, Alasti F, et al. Disease activity in psoriatic arthritis: defining remission and treatment success using the DAPSA-score. Ann Rheum Dis 2015. Published Online First 12 Aug 2015. http://dx.doi.org/10.1136/annrheumdis-2015207507

5 Nell-Duxneuner VP, Stamm TA, Machold KP, et al. Evaluation of the appropriateness of composite disease activity measures for assessment of psoriatic arthritis. Ann Rheum Dis 2010;69:546-9.

6 Aletaha D, Smolen J, Ward MM. Measuring function in rheumatoid arthritis: identifying reversible and irreversible components. Arthritis Rheum 2006;54:2784-92.

7 Smolen JS, Aletaha D, Grisar JC, et al. Estimation of a numerical value for joint damage-related physical disability in rheumatoid arthritis clinical trials. Ann Rheum Dis 2010;69:1058-64.

8 Gladman DD, Mease PJ, Healy P, et al. Outcome measures in psoriatic arthritis. J Rheumatol 2007;34:1159-66.

9 Radner H, Smolen JS, Aletaha D. Impact of comorbidity on physical function in patients with rheumatoid arthritis. Ann Rheum Dis 2010;69:536-41.

10 Radner H, Smolen JS, Aletaha D. Comorbidity affects all domains of physical function and quality of life in patients with rheumatoid arthritis. Rheumatology (Oxford) 2011:50:381-8.

11 Jamnitski A, Symmons D, Peters MJ, et al. Cardiovascular comorbidities in patients with psoriatic arthritis: a systematic review. Ann Rheum Dis 2013:72:211-16

12 Eder L, Thavaneswaran A, Chandran V, et al. Factors explaining the discrepancy between physician and patient global assessment of joint and skin disease activity in psoriatic arthritis patients. Arthritis Care Res (Hoboken) 2015;67:264-72.

13 Smolen JS, Braun J, Dougados M, et al. Treating spondyloarthritis, including ankylosing spondylitis and psoriatic arthritis, to target: recommendations of an international task force. Ann Rheum Dis 2014;73:6-16.

14 Husic $R$, Gretler J, Felber $A$, et al. Disparity between ultrasound and clinical findings in psoriatic arthritis. Ann Rheum Dis 2014;73:1529-36.

15 Salaffi $F$, Ciapetti A, Carotti M, et al. Disease activity in psoriatic arthritis: comparison of the discriminative capacity and construct validity of six composite indices in a real world. Biomed Res Int 2014;2014:528105.

16 Goldsmith $\mathrm{CH}$, Smythe HA, Helewa A. Interpretation and power of a pooled index. J Rheumatol 1993;20:575-8.

17 van der Heijde DM, Van't Hof MA, van Riel PL, et al. Validity of single variables and composite indices for measuring disease activity in rheumatoid arthritis. Ann Rheum Dis 1992;51:177-81.

18 Caperon A, Helliwell PS. Remission in psoriatic arthritis. J Rheumato/ Supp/ 2012;89:19-21.

19 Salaffi F, Ciapetti A, Carotti M, et al. Disease activity in psoriatic arthritis: comparison of the discriminative capacity and construct validity of six composite indices in a real world. Biomed Res Int 2014;2014:528105. 
20 Haroon M, Winchester R, Giles JT, et al. Certain class I HLA alleles and haplotypes implicated in susceptibility play a role in determining specific features of the psoriatic arthritis phenotype. Ann Rheum Dis 2014. Published Online First 26 Sept 2014. http://dx.doi.org/10.1136/annrheumdis-2014-205461

21 FitzGerald O, Haroon M, Giles JT, et al. Concepts of pathogenesis in psoriatic arthritis: genotype determines clinical phenotype. Arthritis Res Ther 2015;17:115.

22 Acosta Felquer ML, Ferreyra GL, Marin J, et al. Remission criteria and activity indices in psoriatic arthritis. Clin Rheumatol 2014;33:1323-30.

23 Eder $L$, Thavaneswaran A, Chandran V, et al. Increased burden of inflammation over time is associated with the extent of atherosclerotic plaques in patients with psoriatic arthritis. Ann Rheum Dis 2015;74:1830-5.
24 Cauli A, Gladman DD, Mathieu A, et al. Patient global assessment in psoriatic arthritis: a multicenter GRAPPA and OMERACT study. I Rheumatol 2011;38:898-903.

25 Coates LC, FitzGerald O, Gladman DD, et al. Results of a randomised controlled trial comparing tight control of early psoriatic arthritis (TICOPA) with standard care: tight control improves outcome. Arthritis Rheum 2013;65:1504-9.

26 Kavanaugh A, Fransen J. Defining remission in psoriatic arthritis. Clin Exp Rheumatol 2006;24(6 Suppl 43):S-83-7.

27 Soriano ER. Defining remission in psoriatic arthritis: are we getting closer? J Rheumatol 2015;42:907-8. 